\title{
Expression of the embryonic morphogen Nodal in cutaneous melanocytic lesions
}

\author{
Limin $\mathrm{Yu}^{1,4}$, Paul W Harms ${ }^{1,4}$, Pedram Pouryazdanparast ${ }^{2}$, David SL Kim ${ }^{1}$, Linglei Ma ${ }^{1,3}$ \\ and Douglas R Fullen ${ }^{1,3}$ \\ ${ }^{1}$ Department of Pathology, University of Michigan, Ann Arbor, Michigan, USA; ${ }^{2}$ Department of Pathology, \\ Northwestern University, Chicago, Illinois, USA and ${ }^{3}$ Department of Dermatology, University of Michigan,
} Ann Arbor, Michigan, USA

\begin{abstract}
Nodal, a potent embryonic morphogen in the transforming growth factor- $\beta$ family, is a proposed key regulator of melanoma tumorigenicity. However, there has been no systematic study of Nodal expression in melanocytic lesions. We investigated Nodal expression by immunohistochemistry in 269 melanocytic lesions, including compound nevi, dysplastic nevi, congenital nevi, Spitz nevi, melanoma in situ, malignant melanoma including the variant desmoplastic melanoma, and metastatic melanoma. We found that the Nodal expression was significantly increased in malignant lesions (including melanoma in situ, malignant melanoma, and metastatic melanoma) compared with compound nevi, Spitz nevi, and dysplastic nevi. Surprisingly, congenital nevi expressed a level of Nodal comparable with malignant lesions, whereas desmoplastic melanoma showed lower expression than nondesmoplastic malignant melanoma $(P<0.05)$. Deep melanoma (Breslow depth $>1 \mathrm{~mm}$ ) displayed a higher percentage of Nodal-positive tumor cells than did superficial melanoma (Breslow depth $\leq 1 \mathrm{~mm}$ ), although there was no statistical difference in the overall staining intensity $(P=0.18)$. Melanomas in situ showed a lower level of Nodal expression than did deep melanomas and metastatic melanomas $(P<0.05)$. The low expression of Nodal in normal and dysplastic nevi, and its increasing expression with the progression of malignant lesions, are suggestive of a role for Nodal in melanoma progression. Modern Pathology (2010) 23, 1209-1214; doi:10.1038/modpathol.2010.101; published online 21 May 2010
\end{abstract}

Keywords: Nodal; transforming growth factor- $\beta$; melanoma; dysplastic nevus

Malignant melanoma accounts for the majority of fatalities from cutaneous cancer and has steadily increased in incidence over the past 30 years. $^{1}$ Melanoma presents challenges to pathologists and clinicians, such as the reliable diagnosis of early/developing lesions and effective treatment of metastatic disease, respectively. ${ }^{2,3}$ A more complete understanding of the molecular changes accompanying melanoma tumorigenesis and progression promises to identify novel diagnostic and therapeutic targets for melanoma.

Nodal is a potent embryonic morphogen in the transforming growth factor (TGF)- $\beta$ superfamily of signaling factors. The Nodal signaling pathway has

Correspondence: Dr D Fullen, MD, Departments of Pathology and Dermatology, University of Michigan, M3261 Medical Sciences I 0602, 1301 Catherine Road, Ann Arbor, MI 48109-0602, USA.

E-mail: dfullen@med.umich.edu

${ }^{4}$ These authors contributed equally to this work.

Received 29 December 2009; revised 4 March 2010; accepted 24 March 2010; published online 21 May 2010 critical roles in vertebrate development, including endoderm/mesoderm specification, anterior-posterior axis positioning, neural patterning, and establishing left-right axis asymmetry. ${ }^{4}$ In vitro, Nodal/ activin signaling maintains the pluripotency of human embryonic stem cells. ${ }^{5-7}$ Although the functions of Nodal in embryogenesis are well known, its potential roles in postnatal physiology and disease are poorly understood. There is extensive evidence that TGF- $\beta$ family signaling has roles both in tumor suppression and tumor progression in malignancies, including melanoma. ${ }^{8,9}$ TGF- $\beta$ has potent antiproliferative effects in normal melanocytes, and this antiproliferative activity is diminished with melanoma progression. ${ }^{10-12}$ It has also been found that TGF- $\beta$ signaling promotes the anchorage-independent growth and metastatic capability of melanoma cells. ${ }^{13,14}$ Consistent with a similar role for Nodal in melanoma aggressiveness, blockade of Nodal signaling in cultured melanoma cells resulted in decreased invasiveness, anchorage-independent growth, and xenograft tumor formation. ${ }^{15}$ 
The initial study of Nodal expression in melanomas supported a role for Nodal signaling in melanomas. Nodal was shown to be expressed in 6 of 10 metastatic melanomas, but only weakly in primary melanomas, and not in the normal skin. ${ }^{15}$ However, the size of this study was limited, and the expression of Nodal in benign and dysplastic nevi was not addressed. The presence or absence of Nodal expression in dysplastic and in situ melanocytic lesions has clear implications for the role Nodal may have in melanoma tumorigenesis and/or progression. In this study, we investigated Nodal expression in a large spectrum of melanocytic lesions.

\section{Materials and methods}

\section{Case Selection}

This research was approved by the Institutional Review Board at the University of Michigan Medical Center. A search of the surgical pathology database was conducted at the Department of Pathology of the University of Michigan to identify melanocytic lesions. A total number of 269 lesions were included in this study, including 29 compound nevi, 28 dysplastic nevi, 30 congenital nevi, 20 Spitz nevi, 24 melanomas in situ, 101 primary malignant melanomas, and 37 metastatic malignant melanomas. Congenital nevi were defined by clinicopathological criteria, including the presence of the lesion at birth. The histological subtypes of malignant melanoma were 23 desmoplastic melanomas, 19 superficial nondesmoplastic malignant melanoma (Breslow depth $\leq 1 \mathrm{~mm}$ ), and 59 deep nondesmoplastic malignant melanoma (Breslow depth $>1 \mathrm{~mm}$ ).

\section{Immunohistochemistry}

For deep melanomas, metastatic melanomas, and desmoplastic melanomas, tissue microarrays were constructed by acquiring $0.6-\mathrm{mm}$ punches (in the case of deep nondesmoplastic malignant melanoma and metastatic malignant melanoma) or $1.0-\mathrm{mm}$ punches (in the case of desmoplastic melanomas) from each formalin-fixed paraffin-embedded archival specimen block. Compound nevi, Spitz nevi, congenital nevi, dysplastic nevi, melanomas in situ, and superficial melanomas were evaluated as individual slides.

For all lesions, formalin-fixed paraffin-embedded $5-\mu \mathrm{m}$ tissue sections were deparaffinized and pretreated with $3 \% \mathrm{H}_{2} \mathrm{O}_{2}$ and Tris-buffered saline. After antigen retrieval, the sections were incubated with mouse monoclonal anti-Nodal antibody (1:100 dilution; ab55676; Abcam, Cambridge, MA, USA) at room temperature for $30 \mathrm{~min}$, followed by EnVision + System horseradish peroxidase (HRP)-conjugated goat anti-mouse (Dako, Carpinteria, CA, USA) for $30 \mathrm{~min}$. Sections were then treated with peroxidase substrate solution containing $0.01 \%$ hydrogen peroxide and $0.05 \%$ DAB (diaminobenzidine tetrahydrochloride, Dako) and counterstained with Harris hematoxylin. Tissue from human endometrial cancer was used as positive control.

Cytoplasmic staining was considered positive for Nodal. Two pathologists (LY and DRF) independently recorded the staining results. The intensity and percentage of positive lesional cells were graded on a scale of $0-3$ ( 0 , no staining; $1,<10 \%$ or weak stain; $2,10-50 \%$ or moderate stain; $3,>50 \%$ or strong stain). The staining index (SI) was calculated as the product of the grades for staining intensity and percentage of Nodal-positive lesional cells.

\section{Statistical Analysis}

Statistical analysis was carried out using StatsDirect software (Version 2.7.2, StatsDirect, Cheshire, UK). The Mann-Whitney $U$-test was used to assess differences in Nodal expression between various melanocytic lesions. Desmoplastic malignant melanoma was excluded from primary malignant melanoma and was analyzed as a separate entity. A $P$-value $<0.05$ was considered statistically significant.

\section{Results}

Results of Nodal immunostaining in melanocytic lesions are summarized in Tables 1 and 2. Epidermal keratinocytes displayed mild nonspecific nuclear staining, which was easily distinguishable from true cytoplasmic staining in melanocytes. Nodal was expressed at low levels in compound nevi, dysplastic nevi, and Spitz nevi (Figure 1a-c), with no significant difference in Nodal expression between these lesions. In nevi with a dermal component, melanocytes in the superficial dermis tended to show stronger Nodal expression (both in terms

Table 1 The expression of Nodal in melanocytic lesions

\begin{tabular}{lcccc}
\hline & $\mathrm{n}$ & $\%^{\mathrm{a}}$ & Intensity & Staining index \\
\hline CN & 29 & 51 & 1.17 & 2.93 \\
DN & 28 & 43 & 1.36 & 3.21 \\
CgN & 30 & 65 & 1.9 & 5.27 \\
SN & 20 & 41 & 1.1 & 2.65 \\
MIS & 24 & 58 & 1.7 & 4.71 \\
MM-S & 19 & 48 & 2.36 & 5.74 \\
DMM & 23 & 46 & 1.52 & 3.78 \\
MM-D & 59 & 71 & 2.25 & 6.49 \\
MMM & 37 & 72 & 2.32 & 6.62 \\
\hline
\end{tabular}

CN, compound nevus; DN, dysplastic nevus; SN, Spitz nevus; $\mathrm{CgN}$, congenital nevus; MIS, melanoma in situ; MM-S, superficial malignant melanoma, Breslow depth $\leq 1 \mathrm{~mm}$; DMM, desmoplastic melanoma; MM-D, deep malignant melanoma, Breslow depth $>1 \mathrm{~mm}$; MMM, metastatic malignant melanoma.

${ }^{\mathrm{a}}$ Percentage refers to the average percentage of Nodal-positive lesional cells.

${ }^{\mathrm{b}}$ Excluded from primary malignant melanomas. 
Table 2 Statistical analysis of Nodal expression (staining index) using the Mann-Whitney $U$-test ( $P$-value)

\begin{tabular}{lcccccccrr}
\hline & $C N$ & $D N$ & $C g N$ & $S N$ & $M I S$ & $M M-S$ & $D M M^{\mathrm{a}}$ & $M M-D$ & $M M M$ \\
\hline CN & - & 0.6445 & $<\mathbf{0 . 0 0 0 1}$ & 0.591 & $\mathbf{0 . 0 0 2 5}$ & $<\mathbf{0 . 0 0 0 1}$ & 0.4234 & $<\mathbf{0 . 0 0 0 1}$ & $<\mathbf{0 . 0 0 0 1}$ \\
DN & 0.6445 & - & $\mathbf{0 . 0 0 0 8}$ & 0.3839 & $\mathbf{0 . 0 2 6 6}$ & $\mathbf{0 . 0 0 0 6}$ & 0.5912 & $<\mathbf{0 . 0 0 0 1}$ & $<\mathbf{0 . 0 0 0 1}$ \\
CgN & $<\mathbf{0 . 0 0 0 1}$ & $\mathbf{0 . 0 0 0 8}$ & - & $\mathbf{0 . 0 0 0 3}$ & 0.3289 & 0.5026 & $\mathbf{0 . 0 0 9}$ & $\mathbf{0 . 0 2 7 3}$ & $\mathbf{0 . 0 1 9 7}$ \\
SN & 0.591 & 0.3839 & $\mathbf{0 . 0 0 0 3}$ & - & $\mathbf{0 . 0 0 8}$ & $\mathbf{0 . 0 0 0 3}$ & 0.2011 & $<\mathbf{0 . 0 0 0 1}$ & $<\mathbf{0 . 0 0 0 1}$ \\
MIS & $\mathbf{0 . 0 0 2 5}$ & $\mathbf{0 . 0 2 6 6}$ & 0.3289 & $\mathbf{0 . 0 0 8}$ & - & 0.1808 & 0.1019 & $\mathbf{0 . 0 0 6}$ & $\mathbf{0 . 0 0 3 8}$ \\
MM-S & $<\mathbf{0 . 0 0 0 1}$ & $\mathbf{0 . 0 0 0 6}$ & 0.5026 & $\mathbf{0 . 0 0 0 3}$ & 0.1808 & - & $\mathbf{0 . 0 0 9 1}$ & 0.1757 & 0.1448 \\
DMM & 0.4234 & 0.5912 & $\mathbf{0 . 0 0 9}$ & 0.2011 & 0.1019 & $\mathbf{0 . 0 0 9 1}$ & - & $\mathbf{0 . 0 0 0 1}$ & $<\mathbf{0 . 0 0 0 1}$ \\
MM-D & $<\mathbf{0 . 0 0 0 1}$ & $<\mathbf{0 . 0 0 0 1}$ & $\mathbf{0 . 0 2 7 3}$ & $<\mathbf{0 . 0 0 0 1}$ & $\mathbf{0 . 0 0 6}$ & 0.1757 & $\mathbf{0 . 0 0 0 1}$ & - & 0.9324 \\
MMM & $<\mathbf{0 . 0 0 0 1}$ & $<\mathbf{0 . 0 0 0 1}$ & $\mathbf{0 . 0 1 9 7}$ & $<\mathbf{0 . 0 0 0 1}$ & $\mathbf{0 . 0 0 3 8}$ & 0.1448 & $<\mathbf{0 . 0 0 0 1}$ & 0.9324 & - \\
\hline
\end{tabular}

CN, compound nevus; DN, dysplastic nevus; SN, Spitz nevus; CgN, congenital nevus; MIS, melanoma in situ; MM-S, superficial malignant melanoma, Breslow depth $\leq 1 \mathrm{~mm}$; DMM, desmoplastic melanoma; MM-D, deep malignant melanoma, Breslow depth $>1$ mm; MMM, metastatic melanoma.

Staining indices for each lesion type were statistically compared using the Mann-Whitney $U$-test ( $P$-value).

${ }^{\mathrm{a}}$ Excluded from primary malignant melanomas.

Bold values indicate statistically significant values $(P<0.05)$.
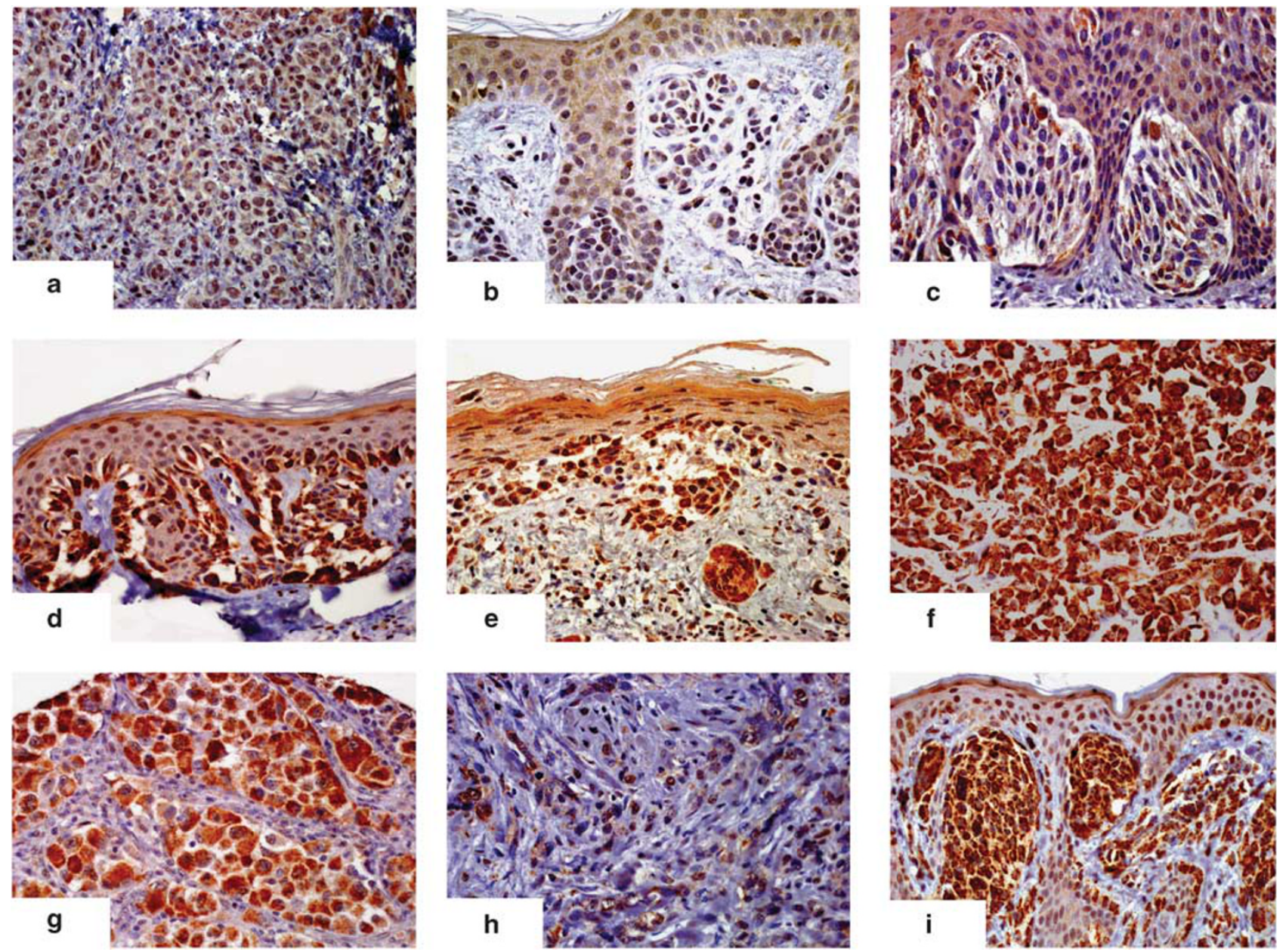

Figure 1 Nodal expression in melanocytic lesions. (a) Nodal expression is weak in compound nevi. (b) Nodal is not overexpressed in dysplastic nevi. (c) Spitz nevi do not express high levels of Nodal. (d) Nodal expression is elevated in melanomas in situ. (e) Nodal is overexpressed in superficial malignant melanomas (Breslow depth $\leq 1 \mathrm{~mm}$ ). (f) Tumor cells of deep malignant melanoma (Breslow depth $>1 \mathrm{~mm}$ ) express high levels of Nodal. (g) Nodal is strongly expressed in metastatic malignant melanomas. (h) Desmoplastic melanomas do not display Nodal overexpression. (i) Nodal is highly expressed in congenital nevi.

of signal intensity and percentage of positive lesional cells) relative to more deeply situated melanocytes. Junctional melanocytes displayed Nodal expression comparable with superficial der- mal melanocytes in the same lesion. Occasional isolated epidermal melanocytes expressed Nodal at an intensity similar to that of junctional melanocytes (not shown). 
Nodal expression was significantly increased in melanomas in situ and primary malignant melanomas compared with compound or dysplastic nevi (Figure 1d-f). In particular, the overall SI for Nodal expression was significantly higher in melanomas in situ relative to dysplastic nevi (4.71 and 3.21 respectively, $P<0.03$ ), although there was no significant difference in the average percentage of Nodal-expressing cells (58 and 43\%, respectively, $P=0.07)$ or staining intensity $(1.70$ and 1.36 , respectively, $P=0.21$ ). In melanomas in situ, malignant cells expressed higher levels of Nodal than did adjacent nevi (Figure 1d). The stratified expression of Nodal observed in benign nevi was not present in superficial malignant melanomas.

To further characterize Nodal expression in melanoma, we compared malignant lesions at different stages of progression. Deep malignant melanomas displayed a higher percentage of Nodal-positive lesional cells than did superficial malignant melanomas (71 and 48\%, respectively, $P<0.003$ ), although the overall SI was comparable (5.74 and 6.49 , respectively, $P=0.18$ ). Nodal expression was increased in deep malignant melanomas and metastatic malignant melanomas (Figure 1g) relative to melanomas in situ (staining indices of 6.49, 6.62, and 4.71, respectively). Desmoplastic malignant melanomas showed no increase in Nodal expression relative to compound nevi (staining indices of 3.78 and 2.93, respectively, $P=0.40$ ) (Figure $1 \mathrm{~h}$ ).

Interestingly, congenital nevi (Figure 1i) showed levels of Nodal that were similar to superficial malignant melanomas (staining indices of 5.27 and 5.74, respectively, $P=0.50$ ), and significantly higher than acquired compound nevi $(P<0.001)$. However, Nodal expression in congenital nevi was lower than in deep malignant melanomas (staining indices of 5.27 and 6.49 , respectively, $P<0.03$ ). In common with other types of nevus, a top-heavy stratified pattern of Nodal expression was observed in the dermal component of congenital nevi. There was no significant association between Nodal SI and nevus size or patient age (data not shown).

\section{Discussion}

Extracellular signaling factors in the TGF- $\beta$ superfamily have diverse roles in embryogenesis and disease. In melanoma and many other malignancies, TGF- $\beta$ signaling inhibits proliferation at early stages, but in late-stage disease promotes tumor progression by increased tumor invasiveness, angiogenesis, and metastasis. ${ }^{9,16}$ Numerous studies have addressed the activities of TGF- $\beta$ in melanoma progression, but less is known regarding the roles of other TGF- $\beta$ family members in melanocytic lesions. Recently, the TGF- $\beta$ family member Nodal emerged as an important signaling molecule in melanoma tumorigenesis and/or progression. ${ }^{15}$ In our large series, we found that Nodal expression was significantly increased in deep malignant melanomas and metastatic melanomas, whereas its expression was low in benign nevi, dysplastic nevi, congenital nevi, Spitz nevi, melanomas in situ, and superficial malignant melanomas. This is the first study to investigate the expression of Nodal in a wide spectrum of melanocytic lesions.

A previous study using immunohistochemistry on formalin-fixed, paraffin-embedded tissues found that Nodal was expressed in metastatic and vertical growth-phase melanoma, but not in normal melanocytes and rarely in radial growth-phase melanoma. ${ }^{15}$ Furthermore, Nodal expression was detected by western blot in aggressive melanoma cell lines but not in cultured benign melanocytes. ${ }^{15,17}$ Similar to the previous report, we detected high levels of Nodal expression in deep malignant melanomas and metastatic malignant melanomas. However, we also observed low levels of Nodal expression in some benign and dysplastic nevi, as well as isolated benign epidermal melanocytes. Our results suggest that the role of Nodal signaling in melanocytic malignancy may be more complex than previously believed. The discrepancy between our observations and those of previous investigators might be due to differences in antibody sensitivity and/or the larger sample size of this study. In addition, Nodal expression has been shown to be sensitive to microenvironmental cues; ${ }^{17}$ hence, Nodal expression patterns in cultured melanocytic cells may differ from in vivo expression. However, our results agree with the impression that Nodal expression increases with melanoma progression.

Interestingly, the level of Nodal expression in congenital nevi was similar to that seen in superficial malignant melanomas. It has been hypothesized that the pathogenesis of congenital nevi may be linked to dysregulation of melanocyte migration, proliferation, or differentiation. ${ }^{18,19}$ Although other members of the TGF- $\beta$ family have been shown to influence melanocyte development/differentiation, ${ }^{20-22}$ a role for Nodal in this process has not been reported. The high expression of Nodal in congenital nevi suggests that Nodal signaling may have as-yet undescribed roles in melanocyte specification, proliferation, migration, and/or differentiation. As Nodal signaling maintains the dedifferentiated phenotype of cultured melanoma cells, ${ }^{15}$ it is possible that Nodal has a physiological role in regulating melanocyte differentiation. Further studies are required to define the specific functions for Nodal in melanocyte development. We did not observe an age-related decline in Nodal expression in congenital nevi; however, as the number of congenital nevi from adult patients was small (4 of 30), a larger sample size may be required to show a difference if it exists.

Desmoplastic melanoma, a rare variant of melanoma consisting of spindled melanocytes within a sclerotic stroma, differs from nondesmoplastic melanoma in morphology, immunohistochemical 
profile, gene expression pattern, and clinical behavior. $^{23,24}$ Tumors are usually positive for S100, but often fail to express other melanocytic markers. In keeping with their distinct molecular profile, desmoplastic melanomas did not display significant Nodal overexpression relative to benign nevi.

The observation that Nodal expression is increased in melanomas raises questions regarding the mechanism(s) by which Nodal signals to melanoma cells and the surrounding stroma. TGF$\beta$ family members, including Nodal, bind and activate type I and type II transmembrane serine/ threonine kinase receptors, which in turn phosphorylate Smad proteins for signal transduction to the nucleus. ${ }^{25}$ In addition, Nodal requires the cellsurface coreceptor Cripto to form a functional signaling complex with the type I receptor ALK4 in most cases. ${ }^{8,26-29}$ Nodal also displays Criptoindependent signaling activities. Nodal signaling through the type I receptor ALK7 is potentiated by, but does not require, the presence of Cripto. ${ }^{27}$ In certain contexts, Nodal-ALK4 signaling might also proceed in the absence of Cripto. ${ }^{30,31}$ In addition, Nodal may block bone morphogenetic protein (BMP) signaling by direct dimerization with the BMP ligand in a Cripto-independent manner. ${ }^{29}$ Nodal signaling in melanomas may be Cripto independent, because only a small sub-population of cultured C8161 melanoma cells express Cripto. ${ }^{32,33}$ Furthermore, melanoma cell lines express TMEFF $1,{ }^{32}$ which has been shown to inhibit Nodal signaling by binding and sequestering Cripto. ${ }^{34}$ Conversely, Lefty and Cerberus, which do not require Cripto to mediate inhibition of Nodal signaling, are not expressed in melanoma cell lines. ${ }^{17,32,35,36}$ Further studies are required to correlate these in vitro findings with patterns of Cripto expression in melanocytic lesions.

In summary, Nodal expression was low in benign and dysplastic melanocytic nevi. Furthermore, Nodal displayed increasing overexpression with melanoma progression. Desmoplastic melanoma did not exhibit Nodal overexpression. Congenital nevi displayed levels of Nodal expression similar to superficial melanoma. Although the precise mechanism(s) by which Nodal signaling in melanoma occurs is yet to be determined, our results strongly support an association between increasing Nodal expression and melanoma progression.

\section{Disclosure/conflict of interest}

The authors declare no conflict of interest.

\section{References}

1 LeBoit PE, (ed). Pathology and Genetics of Skin Tumours. International Agency for Research on Cancer, World Health Organization, International Academy of
Pathology, European Organization for Research on Treatment of Cancer, UniversitätsSpital Zürich. Departement Pathologie. IARC Press: Lyon, 2006, p 355.

2 Busam KJ. The use and application of special techniques in assessing melanocytic tumours. Pathology 2004;36:462-469.

3 Hocker TL, Singh MK, Tsao H. Melanoma genetics and therapeutic approaches in the 21st century: moving from the benchside to the bedside. J Invest Dermatol 2008;128:2575-2595.

4 Schier AF. Nodal signaling in vertebrate development. Annu Rev Cell Dev Biol 2003;19:589-621.

5 James D, Levine AJ, Besser D, et al. TGFbeta/activin/ Nodal signaling is necessary for the maintenance of pluripotency in human embryonic stem cells. Development 2005;132:1273-1282.

6 Vallier L, Alexander M, Pedersen RA. Activin/Nodal and FGF pathways cooperate to maintain pluripotency of human embryonic stem cells. J Cell Sci 2005; 118:4495-4509.

7 Vallier L, Reynolds D, Pedersen RA. Nodal inhibits differentiation of human embryonic stem cells along the neuroectodermal default pathway. Dev Biol 2004; 275:403-421.

8 Bianco C, Adkins HB, Wechselberger C, et al. Cripto-1 activates Nodal- and ALK4-dependent and -independent signaling pathways in mammary epithelial cells. Mol Cell Biol 2002;22:2586-2597.

9 Javelaud D, Alexaki VI, Mauviel A. Transforming growth factor-beta in cutaneous melanoma. Pigment Cell Melanoma Res 2008;21:123-132.

10 Krasagakis K, Kruger-Krasagakes S, Fimmel S, et al. Desensitization of melanoma cells to autocrine TGF-beta isoforms. J Cell Physiol 1999;178:179-187.

11 Rodeck U, Bossler A, Graeven U, et al. Transforming growth factor beta production and responsiveness in normal human melanocytes and melanoma cells. Cancer Res 1994;54:575-581.

12 Krasagakis K, Garbe C, Schrier PI, et al. Paracrine and autocrine regulation of human melanocyte and melanoma cell growth by transforming growth factor beta in vitro. Anticancer Res 1994;14:2565-2571.

13 Javelaud D, Delmas V, Moller M, et al. Stable overexpression of Smad7 in human melanoma cells inhibits their tumorigenicity in vitro and in vivo. Oncogene 2005;24:7624-7629.

14 Javelaud D, Mohammad KS, McKenna CR, et al. Stable overexpression of Smad7 in human melanoma cells impairs bone metastasis. Cancer Res 2007; 67:2317-2324.

15 Topczewska JM, Postovit LM, Margaryan NV, et al. Embryonic and tumorigenic pathways converge via Nodal signaling: role in melanoma aggressiveness. Nat Med 2006;12:925-932.

16 Derynck R, Akhurst RJ, Balmain A. TGF-beta signaling in tumor suppression and cancer progression. Nat Genet 2001;29:117-129.

17 Postovit LM, Margaryan NV, Seftor EA, et al. Human embryonic stem cell microenvironment suppresses the tumorigenic phenotype of aggressive cancer cells. Proc Natl Acad Sci USA 2008;105:4329-4334.

18 Grichnik JM. Melanoma, nevogenesis, and stem cell biology. J Invest Dermatol 2008;128:2365-2380.

19 Kovalyshyn I, Braun R, Marghoob A. Congenital melanocytic naevi. Australas J Dermatol 2009;50: 231-240; quiz 41-2. 
20 Moustakas A. TGF-beta targets PAX3 to control melanocyte differentiation. Dev Cell 2008;15:797-799.

21 Thomas AJ, Erickson CA. The making of a melanocyte: the specification of melanoblasts from the neural crest. Pigment Cell Melanoma Res 2008;21:598-610.

22 Kawakami T, Soma Y, Kawa Y, et al. Transforming growth factor beta1 regulates melanocyte proliferation and differentiation in mouse neural crest cells via stem cell factor/KIT signaling. J Invest Dermatol 2002;118:471-478.

23 Busam KJ. Cutaneous desmoplastic melanoma. Adv Anat Pathol 2005;12:92-102.

24 Busam KJ, Zhao H, Coit DG, et al. Distinction of desmoplastic melanoma from non-desmoplastic melanoma by gene expression profiling. J Invest Dermatol 2005;124:412-418.

25 Shi Y, Massague J. Mechanisms of TGF-beta signaling from cell membrane to the nucleus. Cell 2003;113: $685-700$.

26 Gritsman K, Zhang J, Cheng S, et al. The EGF-CFC protein one-eyed pinhead is essential for Nodal signaling. Cell 1999;97:121-132.

27 Reissmann E, Jornvall H, Blokzijl A, et al. The orphan receptor ALK7 and the Activin receptor ALK4 mediate signaling by Nodal proteins during vertebrate development. Genes Dev 2001;15:2010-2022.

28 Yan YT, Liu JJ, Luo Y, et al. Dual roles of Cripto as a ligand and coreceptor in the Nodal signaling pathway. Mol Cell Biol 2002;22:4439-4449.
29 Yeo C, Whitman M. Nodal signals to Smads through Cripto-dependent and Cripto-independent mechanisms. Mol Cell 2001;7:949-957.

30 Ben-Haim N, Lu C, Guzman-Ayala M, et al. The Nodal precursor acting via activin receptors induces mesoderm by maintaining a source of its convertases and BMP4. Dev Cell 2006;11:313-323.

31 Liguori GL, Borges AC, D’Andrea D, et al. Criptoindependent Nodal signaling promotes positioning of the A-P axis in the early mouse embryo. Dev Biol 2008;315:280-289.

32 Strizzi L, Abbott DE, Salomon DS, et al. Potential for cripto-1 in defining stem cell-like characteristics in human malignant melanoma. Cell Cycle 2008;7:19311935.

33 Strizzi L, Postovit LM, Margaryan NV, et al. Nodal as a biomarker for melanoma progression and a new therapeutic target for clinical intervention. Expert Rev Dermatol 2009;4:67-78.

34 Harms PW, Chang C. Tomoregulin-1 (TMEFF1) inhibits Nodal signaling through direct binding to the Nodal coreceptor Cripto. Genes Dev 2003;17:2624-2629.

35 Chen C, Shen MM. Two modes by which Lefty proteins inhibit Nodal signaling. Curr Biol 2004;14: 618-624.

36 Piccolo S, Agius E, Leyns L, et al. The head inducer Cerberus is a multifunctional antagonist of Nodal, BMP and Wnt signals. Nature 1999;397:707-710. 